\title{
Análise de temperatura de pele da superfície do mar em regiões de alta latitude simulada com o modelo WRF
}

\author{
Simulation of the Sea surface skin temperature in high-latitude regions using the WRF \\ model
}

Alcimoni Nelci Comin e Otávio Costa Acevedo

Universidade Federal de Santa Maria, Santa Maria, RS, Brasil

alcimoni.comin@gmail.com; otavio@ufsm.br

\begin{abstract}
Resumo
Os dados reais de temperatura de superfície do mar (TSM) foram coletados durante o transcecto do navio Polar Almirante Maximiano nas ilhas Shetland do sul e arredores no período 5 a 23 de fevereiro de 2011. Três grades móveis e concêntricas de resolução 12, 3 e $1 \mathrm{~km}$ foram utilizadas nas simulações de temperatura de pele da superfície do mar (TPSM) com o modelo WRF. As grades são deslocadas a cada dia, sempre centradas na posição média do navio (latitude/longitude) durante o transcecto. A TPSM é sempre subestimada em relação a TSM em média $1,5{ }^{\circ} \mathrm{C}$. A velocidade média real do vento observado ao longo do período foi de $8,7 \mathrm{~ms}^{-1}$. Portanto a quantidade de mistura da água do mar entre TSM e TPSM é maior, e a diferença real de temperatura entre as duas camadas fica menor, em média $0,5^{\circ} \mathrm{C}$. A subestimativa do modelo é entorno de $1^{\circ} \mathrm{C}$. Essa subestimativa tem um efeito direto na quantidade de evaporação do oceano para a atmosfera, podendo ocasionar um erro no balanço de energia. A correlação entre os dados foi de 0,84 e raiz do erro médio quadrático 1,87.
\end{abstract}

Palavras-chave: WRF.Arquipélagos Shetland. TPSM.

\begin{abstract}
The in situ data of sea surface temperature (SST) were measured onboard the Polar Ship Almirante Maximiano in the southern Shetland Islands between 5 and 23 February 2011. For the simulations, three concentric nested grids have been used at the $9 \mathrm{~km}, 3 \mathrm{~km}$ and $1 \mathrm{~km}$ spatial resolution in the simulations of the skin sea surface temperature (SSST) with WRF model. The grids are displaced every day, always centered in the middle position of the ship (latitude/longitude) during transect. The SSST is underestimated in comparison with SST on average $1.5{ }^{\circ} \mathrm{C}$. The real average wind speed observed was $8.7 \mathrm{~ms}^{-1}$. Therefore the amount of mixing between SST and SSST is greater, and the temperature difference between the two layers is smaller, on average $0.5{ }^{\circ} \mathrm{C}$. The underestimation of the model is mean $1{ }^{\circ} \mathrm{C}$. This underestimation directly interfere on the amount of ocean evaporation for the atmosphere, which may cause error in the energy balance. The correlation of the SSST with real SST data was 0.84 and root mean square error 1.87 .
\end{abstract}

Keywords: WRF. Shetland islands. SSST. 


\section{Introdução}

A interface atmosfera-oceano se caracteriza por haver um gradiente de temperatura entre uma micro-camada de água do oceano e a atmosfera (Donlon e Robinson, 1997). Esta micro camada de água de espessura de poucos mm é chamada de temperatura de pele da superfície do mar (TPSM). Em geral a TPSM é medida de forma não invasiva usando sensores infravermelho já a temperatura de balde da superfície do mar (TSM) é medida utilizando uma variedade de sensores de imersão em contato. Para muitos propósitos é mais apropriado a TPSM do que a TSM, pois as trocas com a atmosfera ocorre diretamente nesta camada (Dolon et al. 1999). O autor resalta que infelizmente há um número muito reduzido de medidas in situ de TPSM, sendo que a maior parte dos esforços internacionais tem se concentrados para as medidas de TSM.

As ilhas Shetland do Sul são um arquipélago formado por mais de 20 ilhas ao norte e nordeste da Península Antártica, com cerca de $540 \mathrm{~km}$ de extensão e uma área de terra de $3687 \mathrm{~km} 2$. A maior parte dessas ilhas (80-90\%) está coberta de calotas de gelo. $\mathrm{O}$ arquipélago está separado da continente Antártico pelo estreito de Bransfield do Sul e da América do Sul pela passagem de Drake (localização geográfica ver Figura 1).

Não há estudos mostrando simulações com o modelo WRF em altas latitudes envolvente a TPSM. A maioria das simulações para essas regiões são para a velocidade do vento, pressão e temperatura (Powers et al. 2007, Hines et al. 2008, Hines et al. 2011, Bromwich et al. 2013). A pesar de não haver medidas em situ de TPSM o objetivo principal desse estudo é comparar esta variável simulada pelo WRF com medidas reais de TSM. A TPSM é de extrema importância porque afeta diretamente a quantidade de água evaporada para a atmosfera, tendo efeito direto na temperatura e no balanço de energia. Estudos realizados por Bromwich et al. (2013) com o modelo WRF na Antártica, resaltou que “... a atmosfera do modelo é mais seca que o observado, com nebulosidade deficiente, porque as menores temperaturas limitam a sublimação e consequentemente as quantidades de água precipitável sobre a geleira".

\section{Metodologia}

O modelo Weather Research and Forecasting (WRF) foi desenvolvido para a pesquisa, previsão do tempo, modelagem da qualidade do ar, modelagens climáticas em escala regional, pesquisa de furacões entre outras. Suas principais características são: sistema de simulação de dados em 3 dimensões, arquitetura de software que permite a sua execução em paralelo e a extensibilidade do sistema que permite o programa funcionar para diversas escalas de poucos metros até centenas de quilômetros Skamarock et al. (2008). O modelo oferece uma grande diversidade de opções físicas e dinâmicas. Na configuração do modelo utilizado aqui, foram selecionados os seguintes critérios: Esquema microfísico de Thompson et al. (2004) que inclui seis classes de hidrometeoros mais a concentração de gelo como variável prognóstico; parametrização de cumulus Kain (2004) na grades mais externas (na grade interna não foi utilizada parametrização); esquema de rápida transferência radiativa do modelo (RRTM) para a radiação de onda longa, com base em Mlawer et al. (1997); o esquema de Dudhia (1989) para a radiação de onda curta; o esquema de energia cinética turbulenta de MoninObhukov-Janjic (Janjic 2001) para a camada limite planetária; e o esquema Noah de modelo de superfície terrestre (Niu et al. 2011).

Os dados reais de temperatura de superfície do mar (TSM) foram coletados pelo navio Polar Almirante Maximiano da Marinha do Brasil, durante a Operação Antártica 29 no período 5-23 fevereiro de 2011. O navio fez um transcecto nas ilhas Shetland do sul e arredores. Três grades móveis concêntricas e aninhadas, foram utilizadas (Figura 1). As grades são deslocadas a cada dia, sempre centradas na posição média do navio (latitude/longitude) durante o transcecto. O domínio maior tem espaçamento horizontal de $12 \mathrm{~km}$ com 60 pontos em cada direção horizontal. A primeira grade aninhada tem espaçamento horizontal de $3 \mathrm{~km}$ com 141 x 141 pontos. A grade mais interna tem espaçamento horizontal de $1 \mathrm{~km}$, com $187 \times 187$ pontos na direção horizontal. A saída do modelo é a cada 1 hora. Todas as grades utilizam 28 níveis na vertical. As condições iniciais e de contorno do modelo são fornecidas pelo NCEP Final Global (FNL) $1,0^{\circ} \times 1,0^{\circ}$ de resolução 
(http://dss.ucar.edu/datasets/ds083.2/) disponível a cada $6 \mathrm{~h}$.

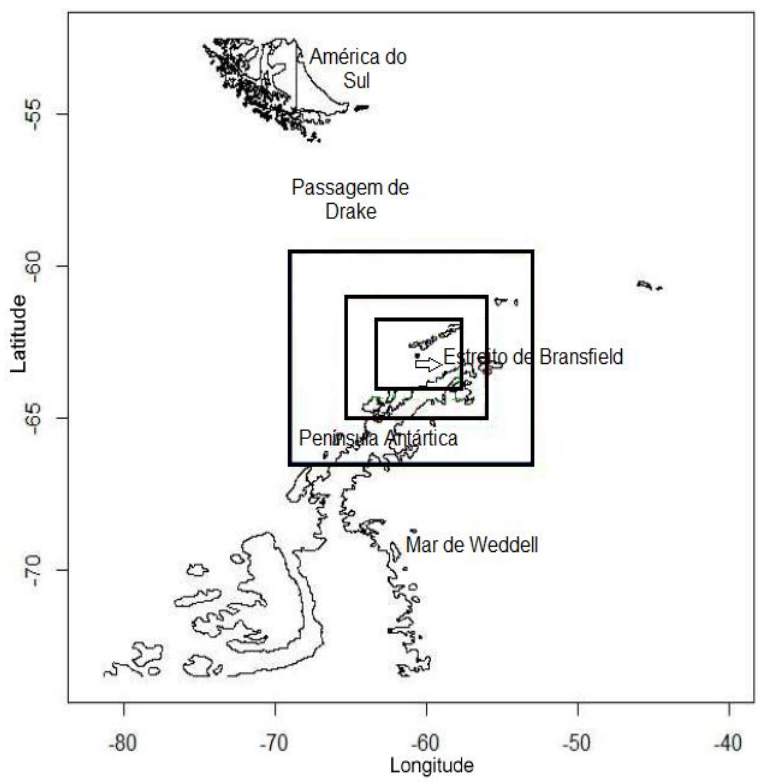

Figura 1 - Localização geográfica do arquipélago das Shetland do Sul (ao norte do estreito de Bransfield), Antártica. Também mostra as 3 grades do WRF, especificamente neste dia centrada na ilha Deception

\section{Resultados e discussão}

A figura 2 mostra aos dados observados de TSM e a TPSM simulados pelo WRF, ambos de frequência de 1 hora. Particularmente no dia 5, observa-se uma temperatura maior em relação os outros dias, pois neste dia o navio estava passando pelo estreito de Drake em direção ao arquipélago das Shetland. Durante a maior parte do período, a TPSM é sempre subestimada em relação a TSM em média $1,5{ }^{\circ} \mathrm{C}$. Dolon et al. (1999) mostraram que, em condições de vento com velocidade menor que $6 \mathrm{~ms}^{-1}$, as diferenças entre o TPSM e TSM comumente atinge $1,5^{\circ} \mathrm{C}$. À noite, esta diferença é menor, atingindo no máximo $0,5{ }^{\circ} \mathrm{C}$. A velocidade média do vento observada ao longo do período estudado foi de $8,7 \mathrm{~m} \mathrm{~s}^{-1}$. Portanto a quantidade de mistura da água do mar entre TSM e TPSM é maior, e a diferença real de temperatura entre as 2 camadas fica menor, em média $0,5{ }^{\circ} \mathrm{C}$. Levando-se em consideração isso a sub estimativa do modelo é entorno de $1{ }^{\circ} \mathrm{C}$. Essa subestimativa tem um efeito sobre a quantidade de evaporação do oceano para a atmosfera e assim, sobre a parametrização de cumulus no WRF. Isso causa uma subestimação dos fluxos de calor latente. Zang e Mcphaden (1995) mostraram que um erro de $1{ }^{\circ} \mathrm{C}$ causa um erro de $10 \mathrm{Wm}^{-2}$ na radiação de onda longa (ROL).

Estudos recentes realizados por Hines et al. (2015), na estação de "SHEBA" no Ártico, para a temperatura de pele "skin" (medida a $2 \mathrm{~m}$ da superfície) utilizando o Polar WRF, encontraram uma subestimativa média de $0,9{ }^{\circ} \mathrm{C}$ em relação aos dados reais no período 16 a 27 de janeiro de 1998. Valores bastante similares entre a TPSM e TSM. Os resultados mostrados aqui sugerem que o WRF subestima a TPSM e, como consequência, há uma menor quantidade de água evaporada e perda de ROL para a atmosfera a partir da superfície do oceano, podendo haver uma superestimativa de radiação de onda curta. Isso pode causar uma subestimativa na temperatura mínima simulada pelo WRF e também um erro no balanço de energia. A correlação entre os dados foi de 0,84 e raiz do erro médio quadrático 1,87. A média simulado de TPSM foi de $0,6{ }^{\circ} \mathrm{C}$, enquanto a média observada de TSM foi $2,1^{\circ} \mathrm{C}$.

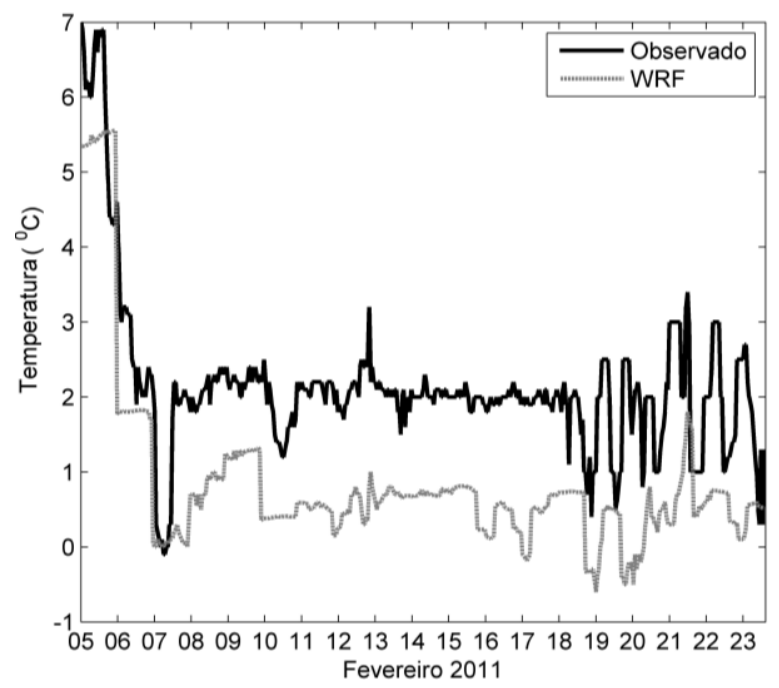

Figura 2 - Dados observados de temperatura da superfície do mar (TSM, linha preta contínua) e os dados simulados pelo WRF para a temperatura de pele da superfície do mar (TPSM, linha cinza tracejada), ambos de frequência de 1 hora

\section{Conclusões}

Há uma tendência de subestimativa entorno de $1{ }^{\circ} \mathrm{C}$ para TPSM simulada pelo WRF em comparação com a temperatura real, isso influencia na taxa de evaporação do oceano para a atmosfera. Consequentemente afeta a previsão de temperatura mínima, em função de haver uma maior perda de energia em forma de ROL. 
Este estudo é de grande importância porque a área em torno da Península Antártica, incluindo o Estreito de Bransfield está se aquecendo mais do que a média global nas últimas décadas (Barrand et al., 2013). As simulações de TPSM por modelos são de grande importância para a compreensão dos efeitos locais destas mudanças.

\section{Agradecimentos}

Agradeço Dr. Ronald Buss de Souza pelo fornecimento dos dados de TSM e ao CNPq/CsF pelo apoio financeiro ao primeiro autor.

\section{Referências}

BARRAND, NE, VAUGHAN DG, STEINER N, TEDESCO M, MUNNEKE PK, VAN DEN BROEKE MR et al. Trends in Antarctic Peninsula surface melting conditions from observations and regional climate modeling. Journal of Geophysical Research. 2013;118:1-16.

BROMWICH DH, OTIENO FO, HINES KM, MANNING KW, SHILO E. Comprehensive 569 evaluation of polar weather research and forecasting performance in the Antarctic. Journal of Geophysical Research. 2013;118:274-292.

DONLON CJ, NIGHTINGALE TJ, SHEASBY T, TURNER J, ROBINSON IS, EMERY WJ. Implications of the oceanic thermal skin temperature deviation at high wind speed. Geophysical Research Letters. 1999;26:2505-2508.

DUDHIA J. Numerical study of convection observed during theWinterMonsoon Experiment using a mesoscale two-dimensional model. Journal of the Atmospheric Sciences. 1989;46: 3077-3107.

HINES KM, BROMWICH DH. Development and testing of Polar WRF. Part I: Greenland Ice Sheet meteorology. Monthly Weather Review. 2008;136:1971-1989.

HINES KM, BROMWICH DH, BAI LS, BARLAGE M, SLATER AG. Development and Testing of Polar WRF. Part III: Arctic Land. Journal of Climate. 2011;24:26-48.
HINES KM, BROMWICH DH, BAI LS, BITZ CM, POWERS JG, MANNING KW. Sea Ice Enhancements to Polar WRF. Monthly Weather Review. 2015;143: 2363-2385.

JANJIC ZI. Nonsingular implementation of the Mellor-Yamada level 2.5 scheme in the NCEP Meso model. NCEP. 2001;437:1-61.

MLAWER EJ, TAUBMAN SJ, BROWN PD, IACONO MJ, CLOUGH SA. Radiative transfer for inhomogeneous atmosphere: RRTM, a validated correlated-k model for the longwave. Journal of Geophysical Research. 1997;102:1666316682.

NIU GY, YANG ZL, MITCHELL KE, CHEN F, EK MB, BARLAGE $M$ et al. The community Noah land surface model with multiparameterization options (Noah-MP): 1 . Model description and evaluation with localscale measurements. Journal of Geophysical Research. 2011;116:1-19.

POWERS JG. Numerical prediction of an Antarctic severe wind event with the Weather Research and Forecasting (WRF) Model. Monthly Weather Review. 2007;135:3134-3157.

SKAMAROCK WC, KLEMP JB, DUDHIA J, GIL DA, BARKER DM, DUDA MG et al. Description of the Advanced Research WRF Version 3. National Center for Atmospheric Research. 2008; Boulder, USA.

THOMPSON G, RASMUSSEN RM, MANNING K. Explicit forecasts of winter precipitation using an improved bulk microphysics scheme. Part I: description and sensitivity analysis. Monthly Weather Review. 2004;132:519-542.

ZANG GJ, MCPHADEN MJ. The relationship between sea surface temperature and latent heat flux in the equatorial Pacific. Journal of Climate. 1995;8: 589-601. 